\title{
Erwachsenwerden in einer unreifen Gesellschaft
}

\section{Fehlende Initiationsrituale als eine Ursache von Jugendproblemen}

PETER MAIER

Peter Maier ist als Lehrer an Gymnasien in Bayern tätig. Neben dem Staatsexamen hat der Autor mehrere Zusatzausbildungen abgeschlossen: zum Gruppenleiter in Themenzentrierter Interaktion, zum Supervisor und zum InitiationsMentor in der Tradition der "School of lost Borders" (Foster/Little). Internet www.initiation-erwachsenwerden.de

\author{
Früher gab es zum Übergang vom Jugendlichen \\ zum Erwachsenen feste Rituale, sei es \\ Konfirmation, Jugendweihe oder der Beginn \\ einer beruflichen Lehre. Ohne solche offiziellen \\ Übergangsrituale besteht die Gefahr, dass sich \\ Jugendlichen eigene, teilweise gefährliche \\ Ersatzrituale suchen oder orientierungslos lange im \\ Lebensabschnitt eines Jugendlichen festhängen.
}

Koma-Saufen, gefährliche Autofahrten, verrückte Mutproben wie »S-BahnSurfen «, Vandalismus, zunehmende Gewalt unter Jugendlichen, Drogen- und Computersucht. Diese Reihe könnte fast beliebig fortgesetzt werden. Aber auch noch ein anderes Phänomen ist bezüglich unserer Jugendlichen heute mehr und mehr zu beobachten: Viele kommen einfach nicht in die »Puschen «, hängen jahrelang orientierungslos herum, scheitern oftmals in der Schule, neigen zur Depression und finden einfach nicht in ihr eigenes Leben, auch wenn sie schon längst volljährig sind. Was ist mit unserer Jugend los?

Wir Eltern, Sozialpädagogen, Lehrer, Psychologen und Politiker stehen diesem Verhalten eines Teils unserer Heranwachsenden im Grunde hilflos gegenüber, auch wenn sich viele Lehrer und manche soziale Einrichtungen mit großem Engagement und hohem finanziellen Aufwand um gestrandete Jugendliche bemühen.

Was bringt Jugendliche dazu, sich aggressiv und gewalttätig zu verhalten? Warum besaufen sich viele von ihnen regelmäßig und glauben, keine Party ohne viel Alkohol bestreiten zu können? Warum sind so viele von ihnen orientierungslos und finden nicht den »Dreh « für ein selbstverantwortliches Leben?

Fragen über Fragen. Sicher kann man eine Reihe von Antworten finden: schlechtes soziales Milieu, gescheiterte Schulkarrieren, überforderte Eltern, unkontrollierter Computerkonsum. Aber keine dieser Ursachen wirkt wirklich als befriedigende Erklärung.

Betrachtet man die ganze Thematik jedoch aus der Perspektive des Initiationsgedankens, so wird vieles verständlich: Jugendliche brauchen geeignete Übergangsrituale - sogenannte »rites of passage « -, um kraftvoll in die Lebensphase des Erwachsenseins eintreten zu können. Doch in unserer Gesellschaft ist das Bewusstsein für Initiation und für Initiationsrituale weitgehend verloren gegangen. Es wird viel mehr der Traum einer " ewigen Jugend « beschworen, die nie erwachsen wird. Andererseits wird von der gleichen Gesellschaft ganz selbstverständlich erwartet, dass junge Menschen erwachsen und voll verantwortlich sind, sobald sie in den Arbeitsprozess, gleich welcher Art, eintreten. Das erscheint mir als großer Widerspruch, der vielfältige Frage aufwirft:

- Was bedeutet eigentlich »Erwachsensein " und wie kann man erwachsenen werden?

- Welche Kriterien kennzeichnet das »Erwachsensein « in unserer Gesellschaft?

- Wie, auf welchem Wege und mit welchen Ritualen und Zeremonien 
können Jugendliche in unserer Gesellschaft den Lebensabschnitt der Jugend verlassen und in die neue Lebensphase des Erwachsenseins hinübergeleitet werden?

- Wohin, besser gesagt "wohinein", also in welches moderne Weltbild und in welches Wertesystem, wollen wir als Gesellschaft unsere Jugendlichen zu Beginn des dritten Jahrtausends eigentlich initiieren?

- Wer soll dies machen, das heißt, wer kann ein »Initiations-Mentor" sein, der die Heranwachsenden bei diesem so wichtigen und würdevollen Übergang mit Rat und Tat zur Seite steht und sie dabei einfühlsam begleitet?

Initiation bedeutet "Eintritt « in eine neue Lebensphase. Alle traditionellen Völker wie beispielsweise die Indianer Nordamerikas hatten ein selbstverständliches Wissen über Notwendigkeit und Bedeutung von »Initiationsritualen«. Nur wenn ihre Jugendlichen, vor allem aber die Jungen, die zukünftigen Männer ihres Stammes, bewusst und mit herausfordernden Prüfungen in das Erwachsensein hinüber geleitet wurden, konnten sie Verantwortung übernehmen und so ihrer Gemeinschaft Schutz gewähren und ihren Fortbestand sichern.

Wir Menschen in westlichen Gesellschaften meinen dagegen, solche Übergangszeremonien seien nicht erforderlich. Welch ein großer Irrtum! Denn dann kann es passieren, dass bereits längst Volljährige in der Tiefe ihrer Psyche nicht wirklich erwachsen geworden sind. Manchmal kann man den Eindruck bekommen, dass selbst 40 - oder 50-Jährige sehr unverantwortlich handeln. Egoismus, Gier, Konsumdenken und die persönliche Vorteilsnahme sind oft die Antriebskräfte von Menschen, die doch eigentlich längst unserer $\mathrm{Ge}$ meinschaft dienen sollten, wenn diese weiterhin gut funktionieren will.

Wie aber sollen dann unsere Jugendlichen, die nicht selten noch idealistisch ausgerichtet sind und etwas für die Gemeinschaft tun wollen, geeignete Vorbilder bei den offiziell Erwachsenen finden, wenn diese in manchen Verhaltensweisen und Haltungen selbst nicht erwachsen sind? Dann trifft leider zu, wovon die Überschrift dieses Beitrags spricht: »Erwachsenwerden in einer unreifen Gesellschaft!«

Nun stellt sich aber die Frage, wie solche Übergangsrituale in unserer heutigen, technisch und medial ausgerichteten Gesellschaft aussehen könnten. Sie sollten ja ansatzweise all die Kriterien enthalten, die zu einem echten Erwachsensein gehören:

- Ablösung von den Eltern

- die Fähigkeit, allein sein zu können

- das Aushaltenkönnen von Entbehrungen und das Bestehen von Ängsten

- Möglichkeiten, seinen Mut und seine Entschlossenheit zu zeigen

- Kontakt zum eigenen Inneren bekommen und die eigene Identität erkennen; seine Stärken und Schwächen sehen und sich selbst annehmen

- Kontakt zum eigenen Familiensystem, zu den Ahnen und zur Geschichte herstellen

- seine eigene Lebensaufgabe, seine eigene Lebensspur, seinen eigenen Beruf erkennen

- Anerkennung durch die Erwachsenen - Initiationszeichen als Ausdruck dafür

Grundsätzlich sehe ich mehrere Wege, wie obige »Kriterien des Erwachsenseins « erworben werden können. Manche Jugendliche haben die Kraft und den Mut, sich alleine auf eine Reise zu machen - weit weg von den Eltern. Ein Gastschul-Aufenthalt während der Schulzeit ist solche eine Möglichkeit, eine Weltreise oder ein freiwilliges soziales Jahr nach der Schulzeit eine andere. Diese äußere Reise kann gleichzeitig zu einer »Heldenreise in die eigene Psyche werden, auf der Ängste zu bestehen sind, die aber zum Schluss den Schatz wirklicher Persönlichkeitsreifung und echten Erwachsenwerdens mit sich bringen.

Eine andere geeignete Möglichkeit ist die "Jugend-Visionssuche«. Dieses Ritual zum Erwachsenwerden, das von erfahrenen Initiations-Mentoren geleitet wird, hat drei Abschnitte: vier Tage Vorbereitung in der Gruppe; drei Tage und drei Nächte "Solozeit« - verlassen der Gemeinschaft; vier Tage Wiedereingliederung in die Gemeinschaft nach der Rückkehr.

In der sogenannten Solozeit geht jeder Jugendliche alleine und ohne Essen in die »Wildnis«; er hat nur einen Rucksack, einen Schlafsack, eine Regenplane und genug Wasser dabei, verzichtet aber auf alle sonstigen Kommunikationsmittel wie Uhr, Handy, MP3-Play- er, Smartphone. Er gilt drei Tage und drei Nächte lang als unsichtbar. Wenn er dann wieder zurückkehrt aus dem Wald, ist er ein anderer Mensch, weil er Ängste durchlitten, das Alleinsein ausgehalten und in der Regel zu sich selbst gefunden hat. Viele Jugendliche sind dabei erwachsen geworden. Dies wird dann von den Leitern und von den angereisten Eltern gewürdigt.

Dieses elftägige Ritual ist jedoch vielen Heranwachsenden zu intensiv, obwohl ich jedem Jugendlichen diese Zeremonie nur bestens empfehlen kann. Daher wurde für Schule und Jugendarbeit eine viertägige Kurzform dieses Rituals entwickelt: der "Walk Away«. Er hat ebenfalls drei Phasen, die Solozeit dauert jedoch nur 24 Stunden. Dennoch ist diese Zeit des Alleinseins für 16- oder 17-jährige Jugendliche Herausforderung genug. Der "WalkAway« ist als Schulveranstaltung, für eine soziale Einrichtung oder für die Jugendarbeit praktisch umsetzbar - ein geeignetes Sicherheitskonzept vorausgesetzt.

Als Gymnasiallehrer habe ich damit bisher seit Jahren ausschließlich gute Erfahrungen gemacht. Die Jugendlichen waren danach mächtig stolz, dass sie den Mut hatten, sich solch einer »Prüfung « zu unterziehen; die angereisten Eltern waren erleichtert und erstaunt, dass sich ihr Sohn oder ihre Tochter zu solch einem Schritt aufraffen und durch all die Anspannungen hindurchgehen konnte. Der persönliche Gewinn daraus war für die Jugendlichen enorm.

Daher sollte die Thematik »Persönlichkeitsentwicklung, Erwachsenwerden, Initiation, Initiationsrituale « in Schulen und in der Jugendarbeit diskutiert werden. Ich wünsche allen Jugendlichen, einen guten Weg zu finden, wie sie ihr Erwachsenwerden bewältigen und dann feiern können.

\section{Literatur}

Peter Maier: Initiation, Erwachsenwerden in einer unreifen Gesellschaft. Band I: Übergangsrituale. Verlag Monsenstein und Vannerdat, Münster 2011. 332 Seiten. 16,50 Euro. ISBN 9783-86991-404-6.

Peter Maier: Initiation. Erwachsenwerden in einer unreifen Gesellschaft. Band II: Heldenreisen. Verlag Monsenstein und Vannerdat, Münster 2011. 342 Seiten. 16,80 Euro. ISBN 978 3-86991-409-1.

www.initiation-erwachsenwerden.de 